\title{
Selective Analysis of Linguistic Features Used in Video Mediated Collaboration: An Indicator of Users Sense of Co-presence
}

\author{
Paulo Melo ${ }^{1}$ and Leila Alem ${ }^{2}$ \\ ${ }^{1}$ LAIV [Interactional Analysis and Videography Lab], \\ Federal University of Pernambuco, Recife, Pernambuco, Brazil \\ ${ }^{2}$ CSIRO ICT Centre, Cnr Pembroke \& Vimiera Roads, \\ Marsfield NSW 2122 Australia \\ paulomelo@gmail.com, leila.alem@csiro.au
}

\begin{abstract}
Studies in video mediated collaboration are going beyond traditional measures of time on task and task accuracy by attempting to qualify specific aspect of users' experience. This paper explores users' sense of co-presence as the extent to which they feel co-present with their partner when building collaboratively a toy over a video conferencing system. A linguistic analysis of the way users are referring to remote objects and places has been conducted in order to investigate the correlation between co-presence score and the frequency of local and remote deixis. Our results indicate that co-presence score is positively correlated to the frequency of local deixis as reported previously [1] and negatively correlated to remote deixis. We conclude that the words used by users when referring to remote objects and places may indicate aspects of user's experience while engaged in remote collaboration.
\end{abstract}

Keywords: Co-presence, CMC, linguistic analysis, Video mediated collaboration.

\section{Introduction}

Traditional video mediated collaboration studies have focused mostly on measures of task performance and user satisfaction. Recent studies in video mediated collaboration research report on measures of user's sense of presence when collaborating with others over a distance. One study [2] reports on the sense of physical presence a specialist doctor experiences when engaged in a remote consultation of a patient. The measure focuses on the extent to which users of video conferencing systems feel physically present in the remote location. Others studies [3, 4] report on the sense of social presence users experience when negotiating with remote partners using a video conference link. In these studies the presence measure focuses on the extent to which users feel connected with their remote partners. One important observation from those studies is the effort to go beyond traditional measures of time on task and task accuracy by attempting to qualify user's sense of presence.

This paper follows this trend and reports on a video mediated collaboration study in which a worker and a helper are assembling jointly a Lego toy. In this study the 
helper's sense of co-presence (i.e. the extent to which they feel in the same room as their remote partner) was assessed. Another work [1] reports an experiment with a similar design where researchers have found that local deixis ${ }^{1}$ and remote deixis ${ }^{2}$ were positively correlated to presence. In that study the sense of presence was measured by a set of questions developed by the authors. We were interested in replicating some aspects of these previous promising findings by this time using a tool based on an existing and established measure of co-presence [5]. In this paper we have used an average measure of co-presence of the whole trial. This average co-presence score and a general measure of the linguistic features were used for investigating potential correlation between co-presence score and linguistic features such as local and remote deixis.

\section{Our Experiment}

Our study was conducted in the context of a more comprehensive experiment which investigated the effects of two different representations of gesture (hand/ pointer) in a remote collaboration on physical task ${ }^{3}$. Thirty-four participants were grouped into randomly assigned 17 pairs of one worker and one helper. Much of the technical setup was based on another work [6]. Each participant faced a standard desktop monitor and had a mat on their desk as the shared workspace. A camera was positioned directly above the mat with a field of view encompassing the entire mat (Figure 1). The Virtual Tea Room technology developed by CSIRO functioned as the technical platform for this experimentation.
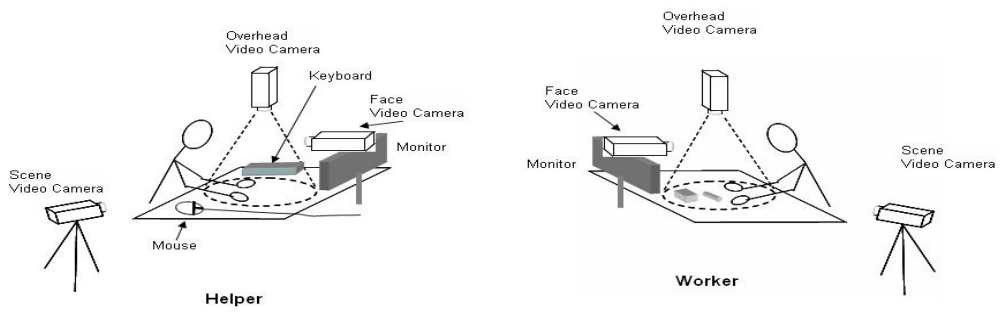

Fig. 1. The technical setup used in the experiment

In this study we have used two methods: a set of subjective measures of copresence and a linguistic analysis based on full transcription of the trials. The sense of co-presence was measured using a set of questions inspired in Schroeder's work [5]. The transcriptions were fed to a text analysis software [7]. The software determined the rate at which the speakers used 70 dimensions of language. Based on [8], we hypothesized that we would find a positive correlation between the frequency of local deixis and co-presence score as established in [1]; and a negative correlation between local deixis and remote deixis as opposed to findings reported [1].

\footnotetext{
${ }^{1}$ Local deixis include words such as: here, this and these.

${ }^{2}$ Remote deixis include words such as: there, that and those.

${ }^{3}$ Initial findings of the more comprehensive study are reported in another paper.
} 


\subsection{Participants and Procedures}

Helpers were asked to guide remotely their partner in assembling the object. While the worker had access to the pieces of Lego toy, the helper had access to the instructions for building the toy. The pair had to built collaboratively the Lego toy under three media conditions administrated in a randomized order. In a final step the pair was asked to assemble the body parts assembled in previous conditions. A post questionnaire was administered after each condition as well as after completion of the final step. A final questionnaire was also administered consisting of demographic information, and preference questions. Then the participants were debriefed and compensated with AU $\$ 40,00$. Each session took approximately 80 minutes.

\section{Results}

An initial analysis indicated a high positive correlation between the co-presence score and the frequency of local deixis, and a negative correlation between presence score and the frequency of remote deixis, and finally, a high negative correlation between local and remote deixis (Table 1). A regression analysis was performed in order to check the linguistic features ability to predict co-presence score. The regression used the frequency of local deixis and the frequency of We category (words as we, our and us). Results indicate that those linguistic variables can predict about $38 \%$ of the whole the co-presence score $(\mathrm{R}$ Square $=.381 ; \mathrm{F}[12,680]=4.31)$.

Table 1. Correlations between local and remote deixis and presence score $(\mathrm{N}=17)$. $* * \mathrm{p}<0.01$; $* \mathrm{p}<0.05$ level (2-tailed).

\begin{tabular}{|c|c|c|c|}
\hline & Remote deixis & Local deixis & Co-presence \\
\hline Remote deixis & 1 & $-.667(* *)$ & -.379 \\
\hline Local deixis & $-.667(* *)$ & 1 & $.581(*)$ \\
\hline Co-presence & -.379 & $.581\left(^{*}\right)$ & 1 \\
\hline
\end{tabular}

\section{Discussion}

In this paper, the use of a co-presence measure developed and used by the presence research community increased our confidence in the interpretation of our findings. Aligned with previous results [1] we have found a high positive correlation between the co-presence score and the frequency of local deixis. In opposition with the same study [1] we also have found a negative correlation between the co-presence score and remote deixis and a negative correlation between the frequency of local deixis and remote deixis. Our results validate what we have hypothesized. It seems reasonable to assume that users during their remote interaction with their partner will either mostly use local deixis (i.e. their linguistic behavior is as if they were co-present with their partner), or mostly use remote deixis (i.e. their linguistic behavior is indicative that they do not feel in the same place as their partner). Users' verbal behavior is hence one indicative of some aspects of their experience while engaged in remote collaboration, more specifically their sense of co-presence. From a methodological 
point of view, our contribution was showing the analysis of specific linguistic features while requiring a lot of effort (full transcription of the trials) is a good candidate method for developing an objective measure of co-presence.

It is proposed to target future work in developing a more cost/effective way of deploying linguistic analysis methods and in exploring relevance of the linguistic approach for measuring/evaluating other aspects of user's experience such as their sense of social presence. Future works will also explore the predictability power of co-presence score through linguistic analysis.

\section{Acknowledgements}

We would like to thank Anja Wessels and Susan Hansen for their help during the data analysis. As well Aiden Wickey, Cara Stitzlein, Alex Krumm-Heller, and Jane Li for their contribution in running the overall experiment. Finally we thank Networking Research Lab at CSIRO for funding this study.

\section{References}

1. Kramer, A., Oh, L., Fussell, S.: Using Linguistic Features to Measure Presence in Computer Mediated Communication. In: CHI 2006 Notes, pp. 913-916. ACM Press, New York (2006)

2. Alem, L., Hansen, S., Li, J.: Evaluating Clinician Experience in a Telemedicine Application: a Presence Perspective. In: Proceedings of OZCHI'06, Sydney, Australia (2006)

3. Stitzlein, C., Alem, L.: When Mixing Physical Presence and Telepresence. In: Proceedings of Presence 06, Cleveland, Ohio, USA (2006)

4. Hauber, J., Regenbrecht, H., Hills, A., Cockburn, A., Billinghurst, M.: Social Presence in Two and Three Dimensional Videoconferencing. In: Proceedings of ISPR (2005)

5. Schroeder, R., Steed, A., Axelsson, A.-S., Heldal, I., Abelin, A., Widestrom, J., Nilsson, A., Slater, M.: Collaborating in Networked Immersive Spaces: as Good as Being There Together? Computer \& Graphics 25, 781-788 (2001)

6. Kirk, D.S., Fraser, D.S., Rodden, T.: The effects of Remote Gesturing on Distance Instruction. In: Proceeding of the International Conference on Computer Supported Collaborative Learning, Taipei, Taiwan, pp. 301-310 (2005)

7. Pennebaker, J.W., Francis, M.E., Booth, R.J.: Linguistic Inquiry and Word Count (LIWC): a Computerized Text Analysis Program. Erlbaum, Mahwah (2001)

8. Gergle, D., Rosé, C., Kraut, R.: Modeling the Impact of Shared Visual Information on Collaborative Reference. In: Proceedings of CHI 2007, pp. 1543-1552. ACM Press, New York (2007) 